\title{
FAST: An extension of the Wavelet Synchrosqueezed Transform
}

\author{
Amey Desai, Thomas C. Richards, and Samit Chakrabarty
}

\begin{abstract}
Extracting frequency domain information from signals usually requires conversion from the time domain using methods such as Fourier, wavelet, or Hilbert transforms. Each method of transformation is subject to a theoretical limit on resolution due to Heisenberg's uncertainty principle. Different methods of transformation approach this limit through different trade-offs in resolution along the frequency and time axes in the frequency domain representation. One of the better and more versatile methods of transformation is the wavelet transform, which makes a closer approach to the limit of resolution using a technique called synchrosqueezing. While this produces clearer results than the conventional wavelet transforms, it does not address a few critical areas. In complex signals that are composed of multiple independent components, frequency domain representation via synchrosqueezed wavelet transformation may show artifacts at the instants where components are not well separated in frequency. These artifacts significantly obscure the frequency distribution. In this paper, we present a technique that improves upon this aspect of the wavelet synchrosqueezed transform and improves resolution of the transformation. This is achieved through bypassing the limit on resolution using multiple sources of information as opposed to a single transform.
\end{abstract}

Index Terms-Wavelet, Filtering, Uncertainty

\section{INTRODUCTION}

A NALYSIS of time-series data follows two main approaches: analysis of amplitude changes with respect to time, and analysis of the frequency content of the signal. Time domain representations reflect changes in the magnitude of the measured quantity with respect to time. Frequency domain representations reflect periodic or repetitive phenomena in the signal with respect to time. Switching between these representations is possible through methods such as the Fourier transform, wavelet transform and others. Data are usually only collected in the time domain. To access frequency domain information, these data must then be transformed from the time representation to the frequency representation.

There are several methods for transformation between time and frequency domain representations. Transformation of purely time domain or purely frequency domain signals is subject to constraints similar to Heisenberg's uncertainty principle that limit both temporal and frequency resolution after transformation. This is because fundamentally, missing information is being estimated. There is a fundamental difference in measurement between time domain and frequency domain. Measuring information about instantaneous frequency, which is basically periodic repetition, requires the evaluation

Amey Desai was with the School of biomedical Sciences, University of Leeds.

Samit Chakrabarty and Thomas Richards are with the University of Leeds. of multiple datapoints, while time domain information is measured in individual datapoints. Improving resolution in frequency requires increasing the number of datapoints. The measured frequency is then attributed to each of these points as opposed to a single point, leading to uncertainty about it's exact point of occurrence. Thus, there is a finite minimum uncertainty when transformations are used.

The Fourier transform, for example, allows for a perfect representation of the total frequency content. In the process, all time domain information is lost. The instantaneous frequency content of the signal cannot be measured. The Fourier transform represents the extreme case whereby the entire signal being analyzed is used for frequency analysis, which results in no temporal resolution but the best possible frequency resolution. The short-time Fourier transform (STFT) divides the signal into overlapping windows, and analyses the frequency content of each window. This approach retains some time domain information, albeit limited by the size of the window. Another technique with improved resolution is the wavelet transform. The wavelet transform approaches frequency analysis differently from the STFT. Instead of dividing the signal into windows, it uses a set of functions with known frequency content, called 'wavelets'. A wavelet is a function composed of sinusoids. It is localized in time. Determining the frequency content of the signal involves comparing (convolving) with the wavelet. The wavelets themselves respond to a range of frequencies surrounding a main 'central' frequency, which can be modified by changing the shape of the wavelet. Synchrosqueezing [1], a scale reassignment technique, allows the transform to improve resolution. Synchrosqueezing is a non-linear operation and exhibits irregular behavior under certain conditions, such as when two concurrent independent components within the signal are close in terms of frequency. [2] quantifies this error and details the factors determining when the error obscures individual distinction of tones. This phenomenon is called 'beating' and involves the two components being identified as a single component with varying frequency. [2] concludes that the bandwidth of the mother wavelet plays an important role in determining when beating occurs. A possible method for mitigating beating is to preprocess the signal using filters that have a smaller bandwidth than the mother wavelet. In this paper, we explore a method which relies on filtering to alleviate beating, called the Filter and Aggregate Synchrosqueezed Transform (FAST).

The following sections explain the wavelet transform and FAST in further detail. 


\section{Elements of the Continuous WaVElet TRANSFORM}

The Continuous Wavelet Transform (CWT) is a versatile technique. The fundamental concept of the wavelet transform is the extraction of frequency domain information using mathematical functions called 'wavelets'. Wavelets are finite length mixtures of several sinusoids. Their finite length makes it possible to extract information about instantaneous frequency distribution. Wavelets are constructed from a set of sinusoids, consisting of a 'central frequency' that has the highest amplitude in the mixture. By modifying this mixture of sinusoids, the wavelet is able to detect different frequencies within the signal. An example of a wavelet can be seen in Figure 1a. The transformation takes place by iteratively convolving the wavelet with the signal at different central frequencies.

The CWT is defined as follows[3]:

$$
S_{w}(a, b)=|a|^{-1 / 2} \int_{-\infty}^{\infty} s(t) \bar{\psi}\left(\frac{t-b}{a}\right) d t
$$

where $\psi(t)$ is a continuous function (mother wavelet), and a $\& \mathrm{~b}$ are scale and translational value parameters, respectively.

There are families of wavelets that each have specific properties designed to enhance particular features of the signal. For example, [4] describes a family of wavelets to be applied to the analysis of seismic signals. We use the Morlet wavelet as an example in the following discussion, but our technique is independent of the wavelet used.

The Morlet Wavelet is defined as follows:

$$
\psi(x)=\left(\left(\pi * F_{b}\right)^{-0.5}\right) e^{2 \pi i F_{c} x} e^{-\left(x^{2}\right) / F_{b}}
$$

where $F_{c}$ is the center frequency and $F_{b}$ is the damping coefficient. Figure 1 depicts an example of the Morlet wavelet.

\section{A. Synschrosqueezing}

Synchrosqueezing is a technique used to sharpen timefrequency transforms. It is applied after the transformation to improve resolution. Synchrosqueezing sharpens transforms by 'reassignment', a process in which parts of the signal containing components which are very similar instantaneously are interpreted as one individual component, resulting in a clearer 'squeezed' depiction of the frequency content. The process is completely reversible and inverse transforms are identical to the original signal.

From the CWT, it extracts the instantaneous frequencies using a phase transform. To sharpen the transform, the instantaneous frequencies are reassigned to a single value in areas where the phase transform is constant. This reduces the spread and results in a sharpened transform. [5] describes the synchrosqueezing procedure in detail. Section III-B1 and equation 4 briefly describe the synchrosqueezing procedure.

Synchrosqueezing requires that individual components of the signal be well separated in frequency to be distinguishable in the synchrosqueezed CWT. Section II-B elaborates upon this aspect of wavelet synchrosqueezed Transform (WSST).
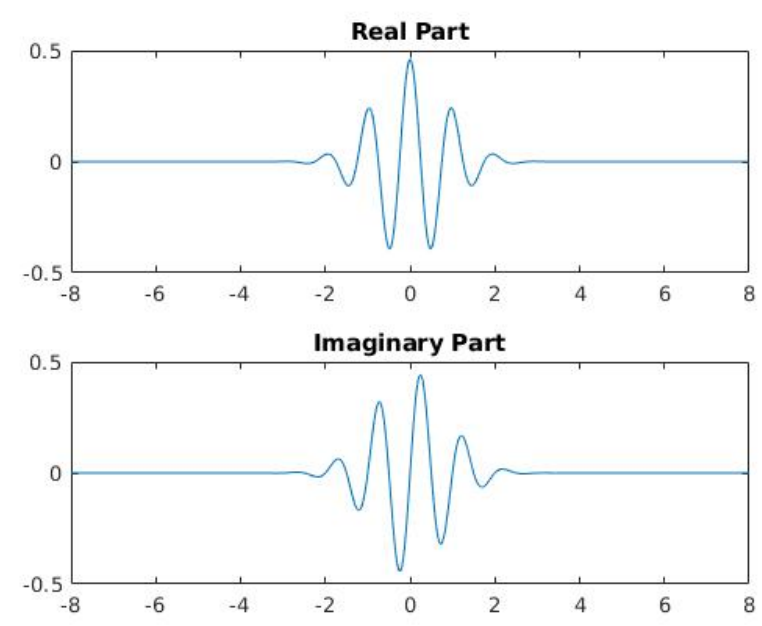

(a) Typical Morlet wavelet: Real and Imaginary Parts

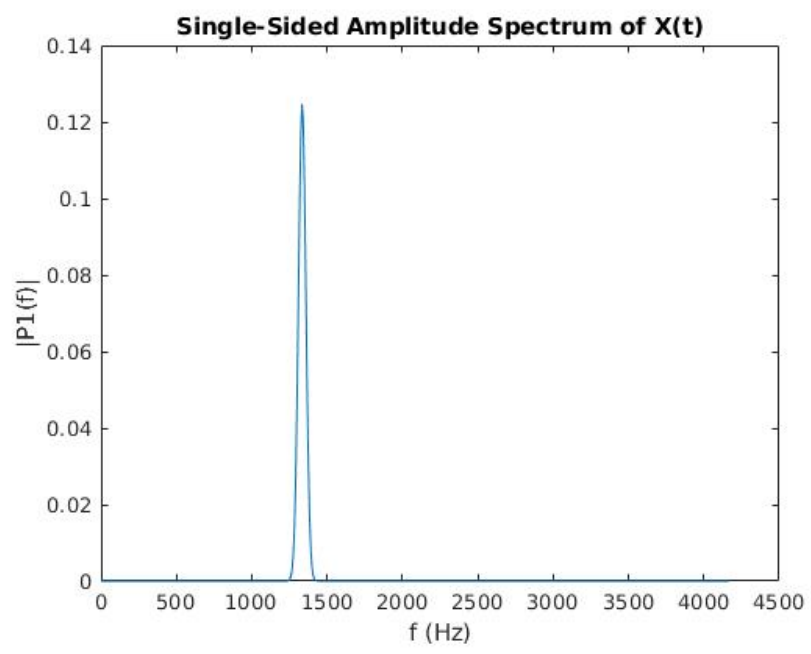

(b) Time and frequency domain representation of a Morlet wavelet

Fig. 1: Morlet wavelet

\section{B. Errors in Synchrosqueezing}

Distinguishing components with close instantaneous frequencies in the synchrosqueezed wavelet transform is a wellstudied problem. [2] have quantified the distortion in the synchrosqueezed spectrum representation. They define an error function e which measures how accurately two such signals can be distinguished by the synchrosqueezed transform. We reproduce their equation for the error here:

$$
e\left(S_{f}, S_{f 1}\right)=\frac{\left.\Sigma_{i, j} \mid\left[\Re S_{f}\left(\xi_{i}, b_{j}\right)=\Re S_{f 1}\left(\xi_{i}, b_{j}\right)\right] \Re S_{(} \xi_{i}, b_{j}\right) \mid}{\Sigma_{i, j}\left|\Re S_{f 1}\left(\xi_{i}, b_{j}\right)\right|^{2}}
$$

where $\left\{\xi_{i}\right\}$ and $\left\{b_{j}\right\}$ are parameters used in calculating the synchrosqueezed transforms $S_{f}$ and $S_{f} 1$.

They conclude that two individual components can be recovered when $\xi \leq \frac{1-\Delta}{1+\Delta}$, where supp $\hat{\psi} \subset[1-\Delta, 1+\Delta]$. Thus, a narrower frequency response of the wavelet $(\Delta)$ is required to improve tone distinction. Below this threshold, the beating phenomenon obscures the true frequency content of the signal. 


\section{Filter \& Aggregate SynchrosqueEZED TRANSFORM (FAST)}

\section{A. Approach}

As detailed in [2], avoiding the 'beating' phenomenon depends on the width support of the mother wavelet. Our approach to this involves restricting bandwidth by iteratively filtering the signal through the desired range and aggregating the resulting transforms. By constraining the filtered signal to a bandwidth narrower than that of the mother wavelet, individual tones can be better distinguished. Further, aggregating multiple instances of the transform help maintain resolution along the time axis by integrating information from multiple sources.

\section{B. Filter and Aggregate}

In order to reduce the misinterpretation of closely spaced tones, we propose a filter-and-integrate approach. The filter and aggregate synchrosqueezed transform (FAST) method involves two major steps: filtering, and transformation.

Given a central frequency $\mathbf{f}$, let $\mathbf{h}$ be a parameter that defines the width of the envelope around the central frequency. The signal is iteratively bandpass filtered in the range $\mathbf{f} \pm \mathbf{h}$, where $\mathbf{h}$ decides the frequency resolution.

Bi-directional filtering is used to avoid phase shifts. The central frequency $\mathrm{f}$ starts at the lower end of the spectrum to be analyzed, and increases by a fixed value $\delta f$ in each iteration. $\delta f$ is always lesser than h, to avoid filter artifacts. The filtered signal is then transformed using the standard WSST. The transforms of each of the filtered signals are then averaged to obtain the aggregate filtered transform. The narrower frequency response of the filter sharpens the frequency resolution but also introduces increased uncertainty in time. This is corrected using the masking step post-processing, which is described in III-C

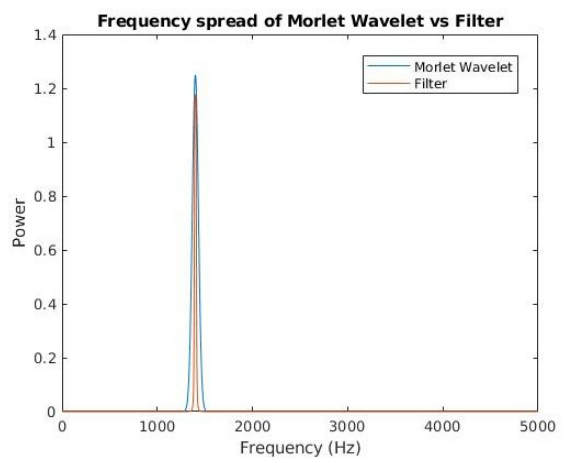

Fig. 2: Comparison between the frequency response of the wavelet and filter

1) Transformation: FAST is equivalent to the CWT integrated over the required frequency range with respect to the passband of the filter.

$$
\begin{gathered}
S_{w}(a, b)=|a|^{-1 / 2} \int_{-\infty}^{\infty} s(t) \bar{\psi}\left(\frac{t-b}{a}\right) d t \\
s(t) \rightarrow s(t) * h(t)
\end{gathered}
$$

$$
\begin{gathered}
\therefore s(t) \rightarrow \int_{-\infty}^{\infty} s(t-\lambda) h(\lambda) d \lambda \\
S_{w}(a, b)=|a|^{-1 / 2} \int_{-\infty}^{\infty}\left[\int_{-\infty}^{\infty} s(t-\lambda) h(\lambda) d \lambda\right] \bar{\psi}\left(\frac{t-b}{a}\right) d t \\
\therefore S_{w}(a, b)=|a|^{-1 / 2} \int_{-\infty}^{\infty}\left[\int_{-\infty}^{\infty} s(t-\lambda) \bar{\psi}\left(\frac{t-b}{a}\right) d t\right] h(\lambda) d \lambda
\end{gathered}
$$

Equation 2 can also be interpret as reducing the width support of the wavelet as follows:

$$
\therefore S_{w}(a, b)=|a|^{-1 / 2} \int_{-\infty}^{\infty} \int_{-\infty}^{\infty} s(t-\lambda)\left[\bar{\psi}\left(\frac{t-b}{a}\right) h(\lambda) d \lambda\right] d t
$$

The synchrosqueezing procedure involves extracting instantaneous frequencies from the CWT output through a phase transform $\omega_{f}$. It is proportional to the partial derivative of the CWT with respect to translation, divided by $2 \pi i S_{w}(a, b)$. The phase transform is as follows:

$$
\omega_{f}(a, b)=\frac{\partial b S_{w}(a, b)}{2 \pi i S_{w}(a, b)}
$$

Following this, the CWT is squeezed over regions where the phase transform is constant. All values from the region are reassigned to the centroid of the region to sharpen output from the CWT. Since the filtering is bidirectional and does not cause a phase shift, synchrosqueezing is unaffected by FAST.

2) Reconstruction:

$$
s(t)=C_{\psi}^{-1} \int_{-\infty}^{\infty} \int_{-\infty}^{\infty} S_{w}(a, b)|a|^{-1 / 2} \bar{\psi}\left(\frac{t-b}{a}\right) d b \frac{d a}{a^{2}}
$$

where $C_{\psi}=\int_{-\infty}^{\infty} \frac{\overline{\hat{\psi}}(\omega) \hat{\tilde{\psi}}(\omega)}{|\omega|} d \omega C_{\psi}$ is the admissible constant.

$$
\begin{aligned}
& s(t)=C_{\psi}^{-1} \int_{-\infty}^{\infty} \int_{-\infty}^{\infty}\left\{|a|^{-1 / 2} \int_{-\infty}^{\infty}\right. \\
& \left.\left[\int_{-\infty}^{\infty} s(t-\lambda) \bar{\psi}\left(\frac{t-b}{a}\right) d t\right] h(\lambda) d \lambda\right\}|a|^{-1 / 2} \bar{\psi}\left(\frac{t-b}{a}\right) d b \frac{d a}{a^{2}} \\
& s(t)=C_{\psi}^{-1} \int_{-\infty}^{\infty} \int_{-\infty}^{\infty}\left\{|a|^{-1 / 2} \int_{-\infty}^{\infty}\right. \\
& \left.\left[\int_{-\infty}^{\infty} s(t-\lambda) \bar{\psi}\left(\frac{t-b}{a}\right) d t\right]\right\}|a|^{-1 / 2} \bar{\psi}\left(\frac{t-b}{a}\right) d b \frac{d a}{a^{2}} h(\lambda) d \lambda \\
& s(t)=C_{\psi}^{-1} \int_{-\infty}^{\infty}\left\{\int_{-\infty}^{\infty} \hat{S_{w}}(a, b)|a|^{-1 / 2} \bar{\psi}\left(\frac{t-b}{a}\right) d b \frac{d a}{a^{2}}\right\}
\end{aligned}
$$

Inverse FAST, similar to inverse WSST does not retain the mean of the data. Inverse FAST also has a different amplitude scale as compared to inverse WSST due to the iterative addition of successive WSSTs. The data is retrieved exactly apart from these factors. 


\section{Masking}

Filtering improves frequency resolution but reduces temporal accuracy. To overcome this, we apply Masking. In order to retain time axis accuracy, we create a mask from the original WSST response. The mask includes areas of the WSST where the response magnitude is greater than a certain threshold $\tau$, typically the mean of the power in WSST. The result of the filtered and aggregated transform is then multiplied elementwise with the mask. This combines the time resolution of the original WSST with the improved frequency resolution after filtering.

Since the transform has the highest magnitude precisely in the areas that correspond most closely to the central frequencies of the wavelet, it might appear as though simply thresholding the WSST representation would be equivalent. However, the beating pattern observed in the transform is a fundamental misinterpretation of the signal and cannot be separated by thresholding. These effects are described and demonstrated visually in the discussion.

\section{Summary}

To summarize, the algorithm for analyzing from $\mathrm{f} 1$ to $\mathrm{f} 2$ is as follows:

- For i from f1 to $\mathrm{f} 2$ (in steps of $\delta f$ )

Construct a 4th order Butterworth bandpass filter centered on $\mathrm{i}$ with a $-3 \mathrm{~dB}$ bandwidth of $\mathbf{2 h} \mathbf{H z}$

Zero-phase filter signal

Obtain wavelet transform of filtered signal

- Average transforms from previous step to get filtered transform W

- Threshold original WSST result to create mask M

- Element-wise multiplication of $\mathbf{M}$ with $\mathbf{W}$

\section{Discussion}

In this paper, we propose the FAST methodology, a timefrequency transformation technique that builds upon the WSST methodology through iterative filtering in order to improve frequency resolution for signals with independent components that may at time become close in frequency. FAST shows superior frequency resolution than WSST, especially when the concurrent components are close in frequency. In signals processed with FAST, beating phenomena are suppressed to an extent which cannot be achieved with WSST.

Using synthetic and real-world examples, in the following paragraphs, we describe and show visual examples of the advantages and applications of the FAST technique.

To demonstrate the application of FAST for resolving common problems in time-frequency domain data, we generated two synthetic signals containing different types of superimposed frequency overlap (Figure 3 ). The first synthetic signal (Figure 3, left column) is highly susceptible to beating (see frequencies below $400 \mathrm{~Hz}$ in WSST) since it is composed of different components which are close in terms of frequency. The second synthetic signal (Figure 3, right column) is composed of transient intermittent components of constant amplitude at different frequencies, which show inconsistency in amplitude and displays artifacts at the termination of transient signals with WSST. The ideal spectra of the example signals are shown in Figure $3 \mathrm{a}$ and $\mathrm{b}$, respectively. For comparison, we then show the transform of each signal with WSST (Figure 3c, d) and FAST (Figure 3e, f). FAST (Figure 3e, f) is superior for distinguishing individual components, and is significantly better at accurately resolving the signal without artifacts, as exhibited by a more similar spectrogram to the actual spectra (Figure 3a, b) than the WSST (Figure 3c, d).

We further explored the utility of FAST in comparison to WSST by transforming both a step function and a quadratic chirp with each technique. The ideal spectra of the step function and quadratic chirps are shown in Figure $4 a$ and $b$, respectively. These signals show how different phenomena are resolved in time-frequency spectra, the step function for a step in frequency, and the quadratic chirps for two close frequency components which progressively decrease in frequency over time to span the whole frequency range. Whilst FAST shows better frequency resolution without artifacts at the step (Figure 4c), filter artifacts mean that the signal amplitude is not constant (Figure 4e). Whilst only one chirp was visible with WSST (Figure 4d), FAST made it possible to distinguish the two independent chirps (Figure 4f). This example is particularly challenging for time-frequency transformations, because the independent components are not uniformly separated in terms of frequency throughout the duration of the signal.

Finally, we compared WSST to FAST using real-world data. We used accelerometer data from a vibration motor (Figure 5a, b), which replicates real-world applications such as checking the consistency/function of a manufacturing unit where alterations in the frequency content could indicate faults. The motor is run on an input voltage that is stepped-up from $1.2 \mathrm{~V}$ to $3 \mathrm{~V}$ and then back, held at each voltage level for a few seconds. The FAST transformation (Figure 5c) has better signal to noise ratio WSST (Figure 5d). The inverse of FAST (Figure 5e) is perfectly correlated with the inverse of WSST (Figure 5f), demonstrating the invertibilty of FAST. Note that the inverse of FAST is scaled in amplitude compared to WSST due to the aggregation of filtered transforms, but is otherwise identical to the inverse WSST. 


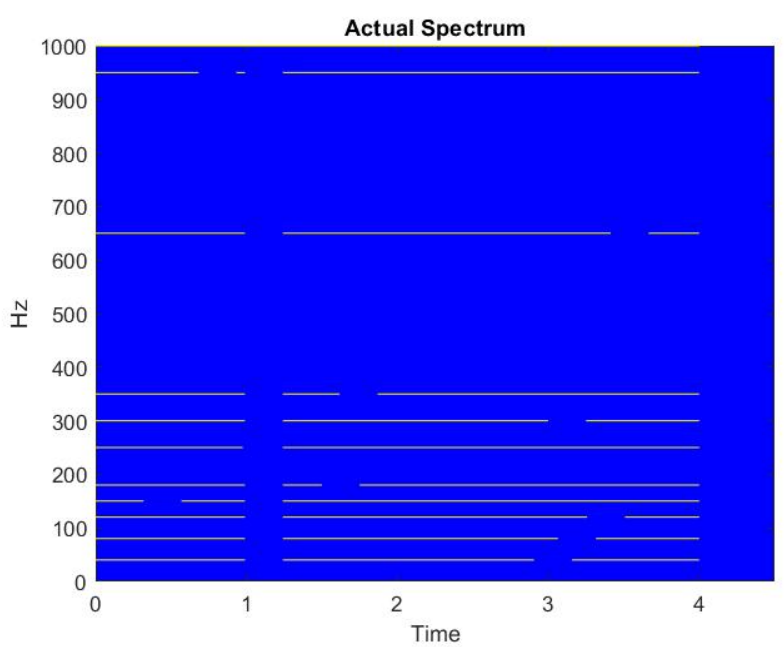

(a) Actual Spectrum 1

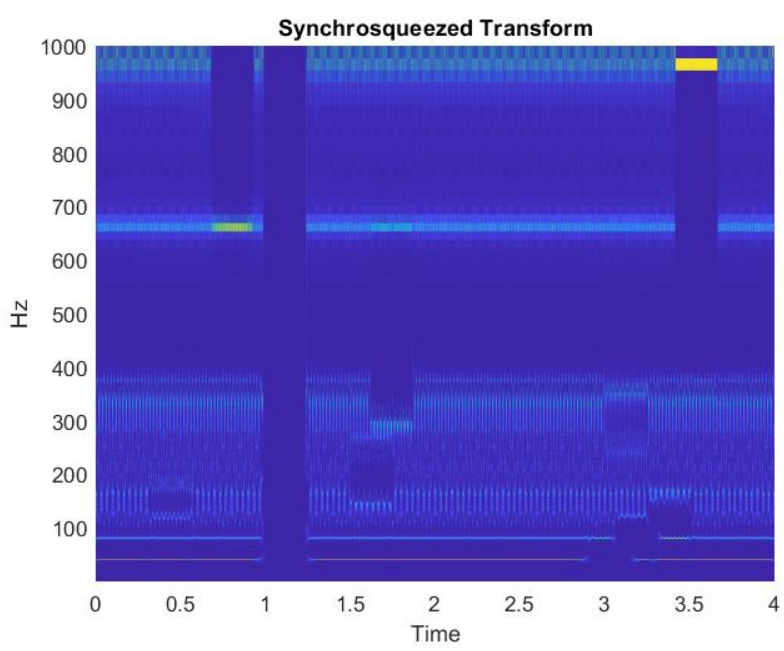

(c) WSST 1

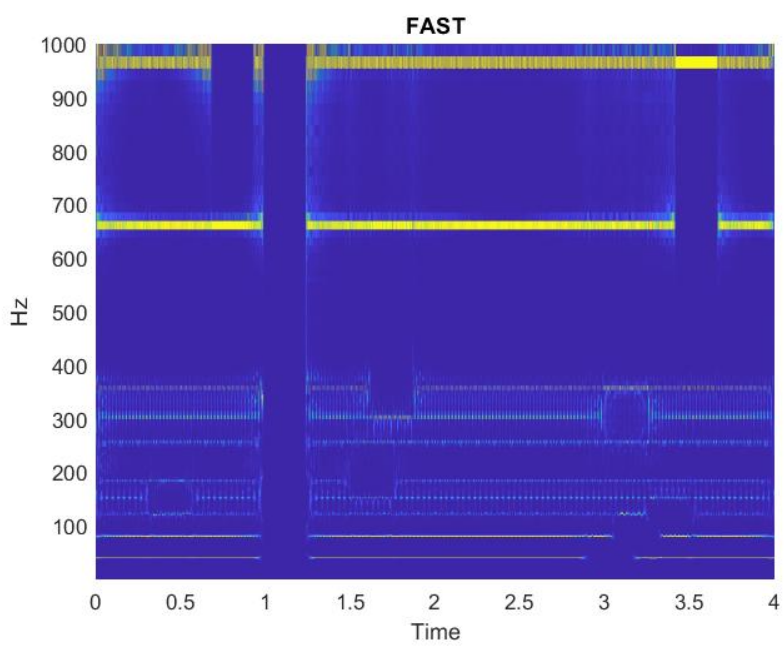

(e) FAST 1

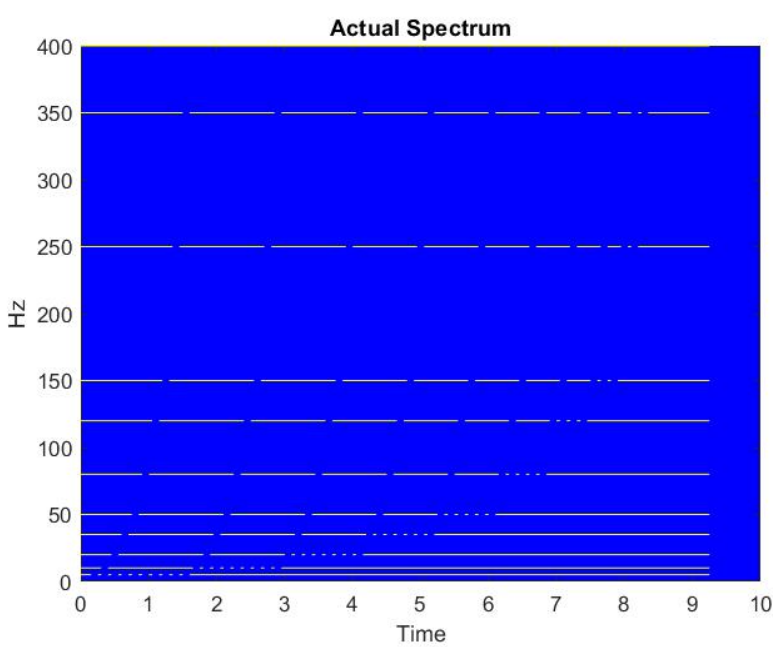

(b) Actual Spectrum 2

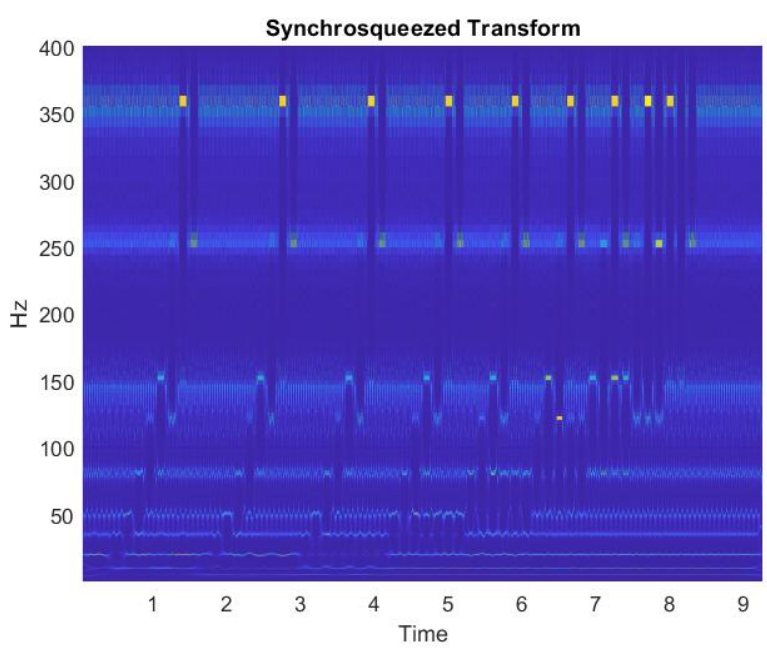

(d) WSST 2

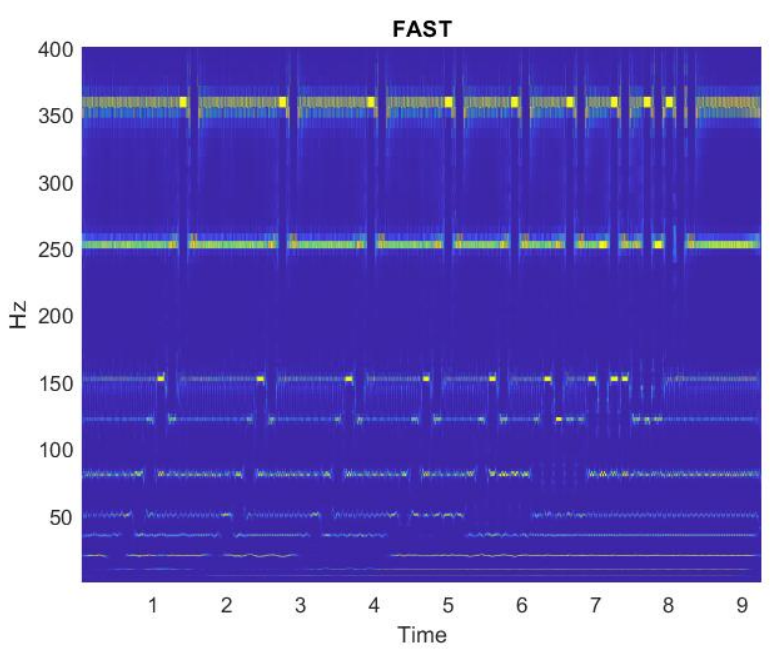

(f) FAST 2

Fig. 3: A comparison of FAST and WSST on synthetic signals with abrupt changes

Two examples (left column, right column) showing representations of the actual spectra of two signals $(a, b)$ demonstrating the better separation of frequency components with FAST (e, f) compared to WSST (c)(d) in time-frequency transformations of multi-component signals. 


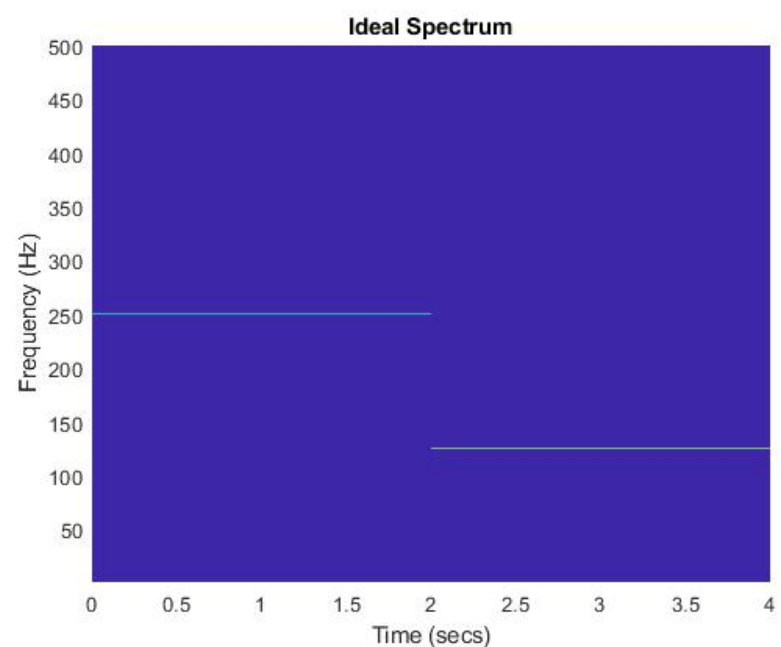

(a) Ideal Spectrum of a step function

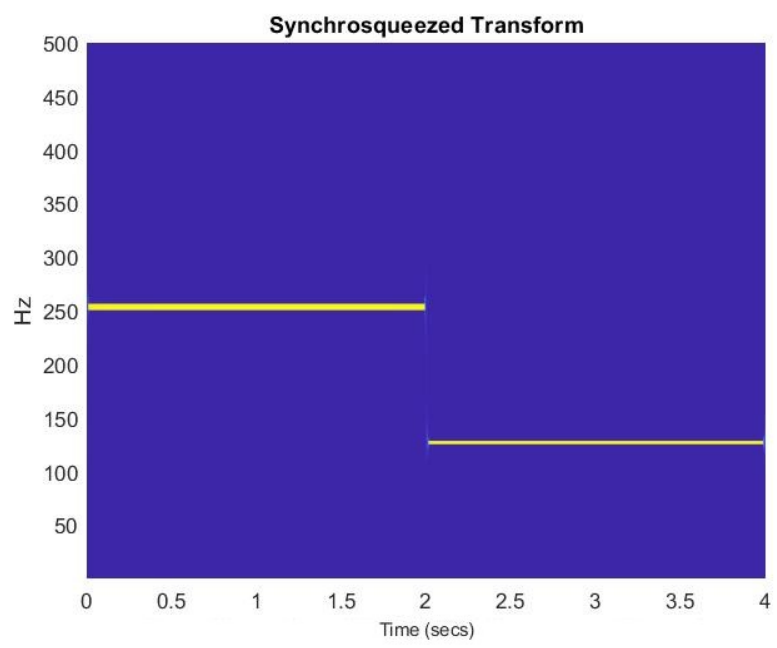

(c) WSST of a step function

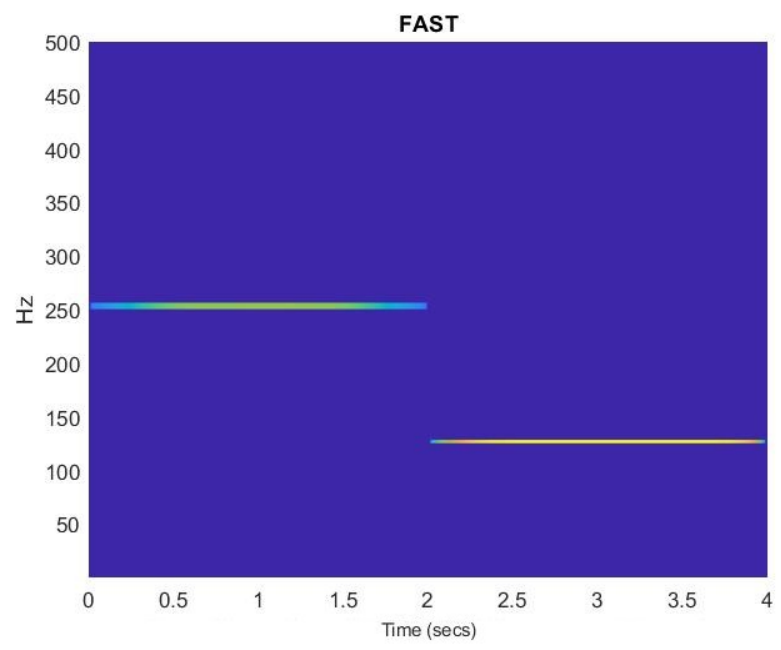

(e) FAST of a step function

Fig. 4: Comparing performance in some well known signals. Left column: Comparison between WSST and FAST transformations of a step function (in the frequency domain), showing the ideal spectra (a). FAST (e) has less interference from beating compared to WSST (c) at the point of transition in frequency. Right column: Comparison of transformations of two quadratic chirps, close in frequency, showing the ideal spectra in (b). Individual components are not distinguishable in WSST (d) while they are better separated in FAST (e).

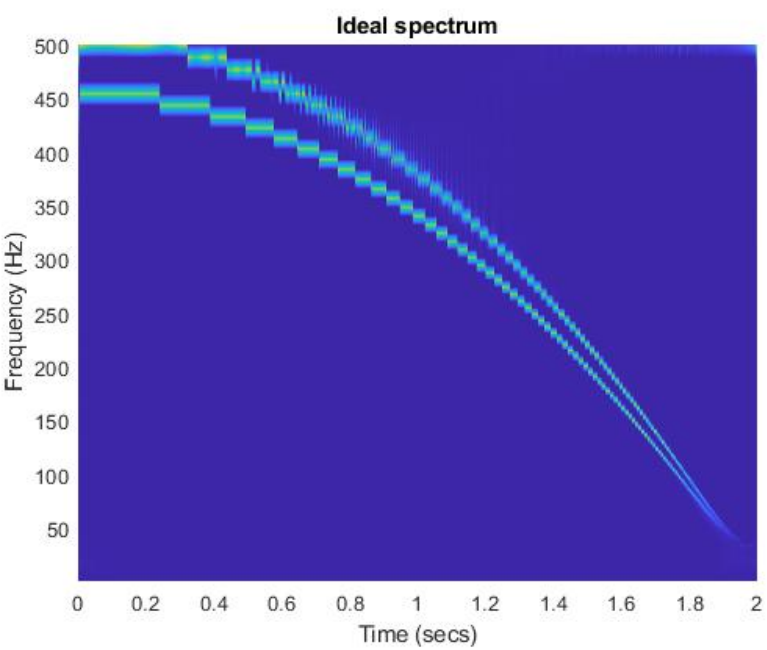

(b) Ideal Spectrum two close quadratic chirps

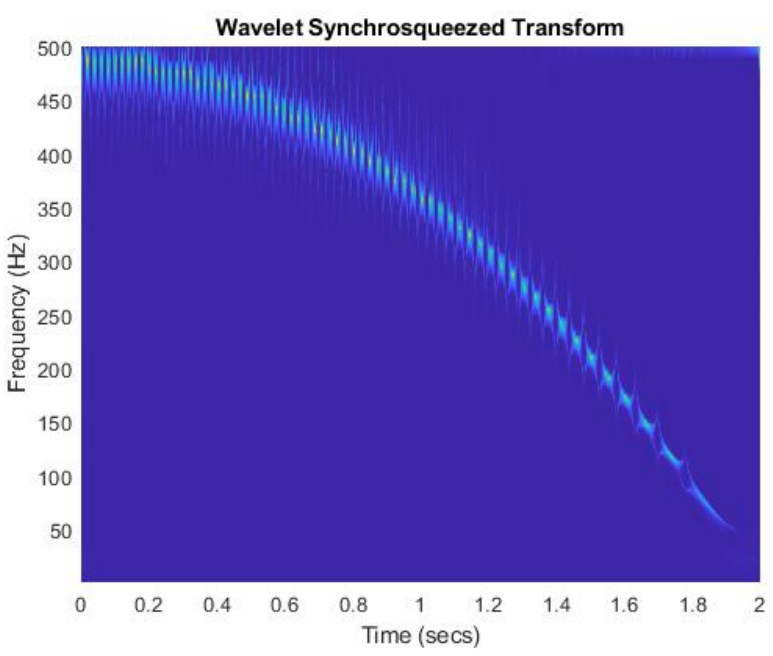

(d) WSST of two close quadratic chirps

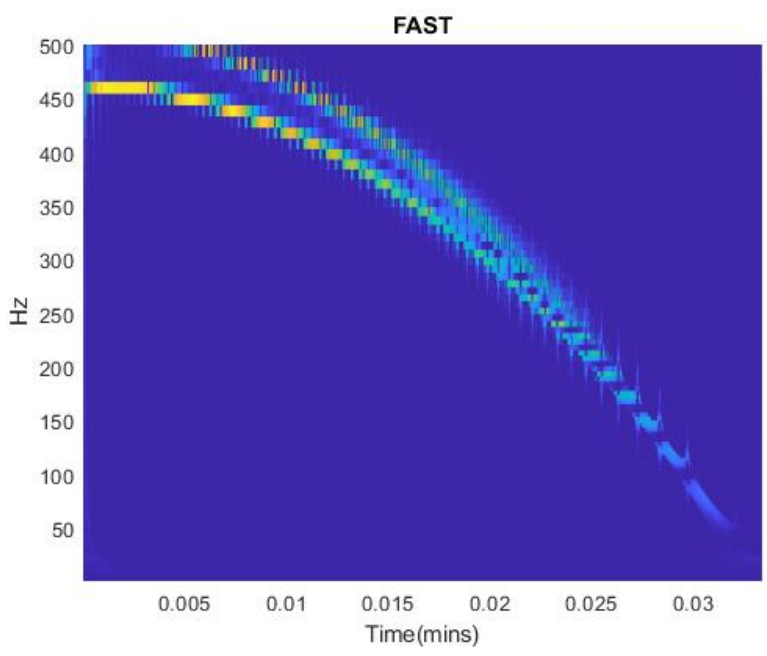

(f) FAST of two close quadratic chirps 


\section{CONCLUSION}

Wavelet transform is a popular method for analyzing the frequency content of signals. It is widely implemented and offers a convenient and powerful tool for time-frequency analysis. Synchrosqueezing is a technique used in the wavelet transform to improve resolution. However, there are specific shortfalls of synchrosqueezed wavelet transforms in the frequency domain such as beating and artifacts which require refinement of the technique. Independent components with instantaneously similar frequencies are often indistinguishable at such instants when transformed to the time-frequency domain using the synchrosqueezed wavelet transform. Wavelet transform involves convolving the signal with a 'wavelet'. The synchrosqueezing technique may not be able to distinguish parts of independent components of the signal that are close in frequency. With a single transform, there is an inherent limit for the maximum resolution that can be obtained. We developed FAST to address these issues and increase the applicability and scope of the synchrosqueezed transform. With FAST, it is possible to better distinguish components with similar frequencies than with the WSST in the frequency domain, by using multiple sources of information (multiple filtered transforms) before applying time-frequency transformation.

FAST increases frequency resolution, and maintains resolution in the time domain. With careful wavelet design, what we achieved with FAST, and improvements in the time-domain can be achieved more elegantly. However, designing wavelets requires technical expertise which may not be accessible in many environments where we envision FAST to be useful. We intend FAST to be a bridge until accessible tools for wavelet development are available. A limitation of FAST is that it is slower than traditional time-frequency transformation algorithms due to its iterative nature. Parallel processing and better targeted filtering may be used to improve processing speed in FAST.

FAST is an accessible and easy to apply process that shows significant improvements on currently available wavelet transform methods such as WSST for avoiding artifacts and beating. However, the computational processing time remains a limitation which should be addressed in the future.

Code and results for FAST are available at https://github.com/sc808/FAST.

\section{ACKNOWLEDGMENT}

The authors would like to thank Mr Rafael de Castro Aguiar and Dr Ioannis Delis from the University of Leeds and Dr. Edward Jero from Vellore Institute of Technology for their feedback during manuscript preparation.

\section{REFERENCES}

[1] I. Daubechies, J. Lu, and H.-T. Wu, "Synchrosqueezed wavelet transforms: An empirical mode decomposition-like tool," Applied and computational harmonic analysis, vol. 30, no. 2, pp. 243-261, 2011.

[2] H.-T. Wu, P. Flandrin, and I. Daubechies, "One or two frequencies? the synchrosqueezing answers," Advances in Adaptive Data Analysis, vol. 03, no. 01n02, pp. 29-39, 2011. [Online]. Available: https://doi.org/10.1142/S179353691100074X
[3] I. Daubechies, "Orthonormal bases of compactly supported wavelets," Communications on pure and applied mathematics, vol. 41, no. 7, pp. 909-996, 1988.

[4] P. Goupillaud, A. Grossmann, and J. Morlet, "Cycle-octave and related transforms in seismic signal analysis," Geoexploration, vol. 23, no. 1, pp. 85-102, 1984.

[5] S. Meignen, T. Oberlin, and D.-H. Pham, "Synchrosqueezing transforms: From low- to high-frequency modulations and perspectives," Comptes Rendus Physique, vol. 20, no. 5, pp. 449-460, 2019, fourier and the science of today / Fourier et la science d'aujourd'hui. [Online]. Available: https://www.sciencedirect.com/science/article/pii/S163107051930101X

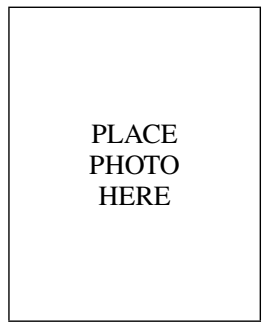

Samit Chakrabarty https://orcid.org/0000-00034688-1459

Samit Chakrabarty has been studying the role of spinal circuits in execution of motor tasks, their modulation by peripheral sensory and descending inputs from the brain, focussing on the plastic changes that the system undertakes during development or disease. After receiving his BSc in Zoology, Biochemistry from St Xavier's College, Mumbai he pursued a PhD in Neurophysiology of the mammalian spinal cord at the University of Cambridge, UK. This was then followed by postdoctoral training at Columbia University, NYC and University of Manitoba, Winnipeg. He has since moved to University of Leeds as an academic researcher and is active in the feld of sensory and motor control, rehabilitation and use of technology to both study and better the lives of those with maladies afecting sensory and motor function.

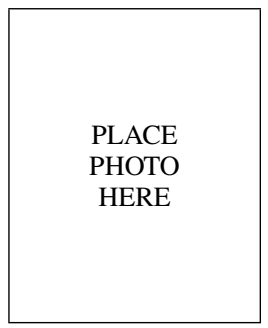

Amey Desai https://orcid.org/0000-0003-2881-9336

Amey Desai received his BE in Electrical and Electronics Engineering from BITS Pilani University, India in 2018. His research interests revolve around neurophysiology and applications in rehabilitative and preventive therapies.

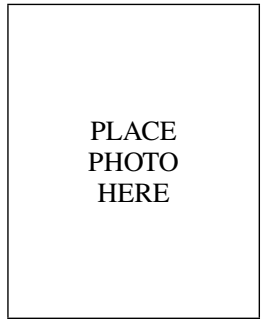

Biography text here.
Thomas C. Richards https://orcid.org/0000-00024447-4334

THOMAS CHRISTOPHER RICHARDS received a BSc in Medical Sciences from the University of Leeds, UK, in 2014. He then went on to study a $\mathrm{Ph} . \mathrm{D}$. in Neurophysiology in the laboratory of $\mathrm{Dr}$ Samit Chakrabarty at the University of Leeds, before working at Leeds Institute of Data Analytics. His research interests are human reflex pathways, muscle synergies, and using data science approaches to real word problems. 


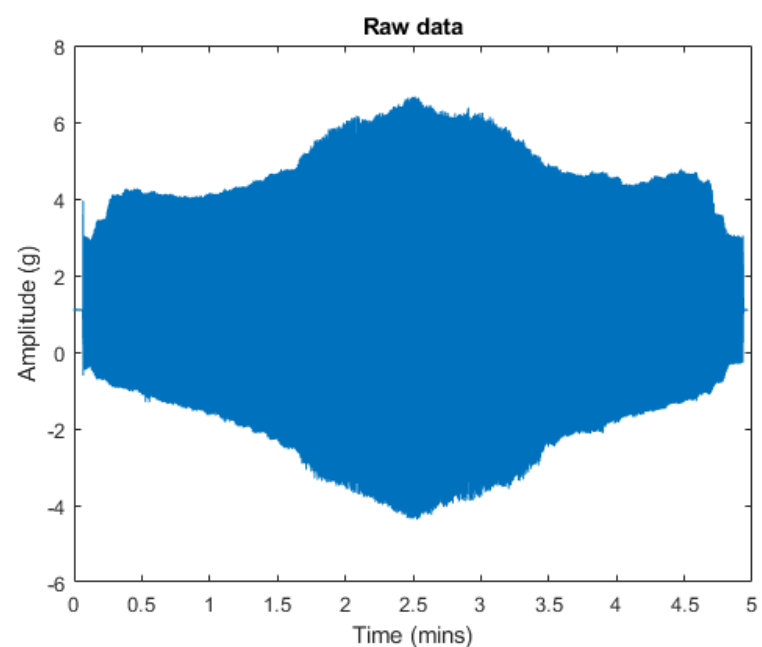

(a) Raw Data

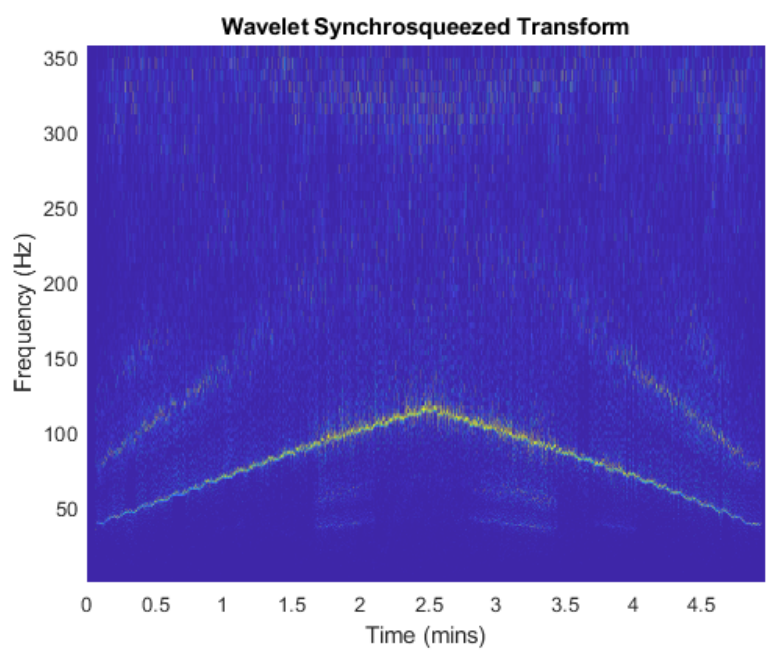

(c) WSST

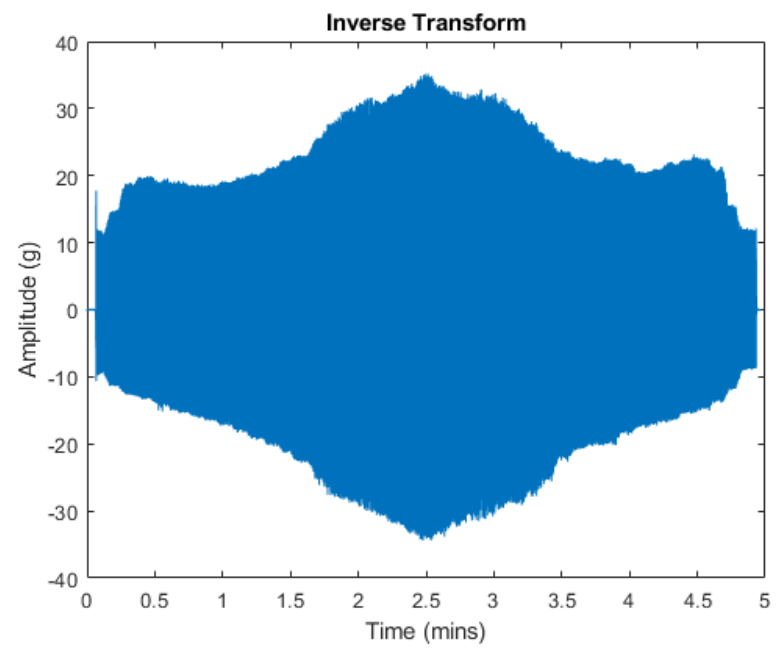

(e) Inverse FAST

Fig. 5: Real World motor vibration example

A real world illustration of FAST. Accelerometer data from a vibration motor was sampled at $75 \mathrm{~Hz}$ (a), and an expanded section to show detail (b). FAST (d) has lower noise and fewer beating artifacts compared to WSST (c). The inverse transform of FAST is scaled as compared to the original data, but otherwise identical (e). The inverse transforms of WSST and FAST are perfectly positively correlated (f).

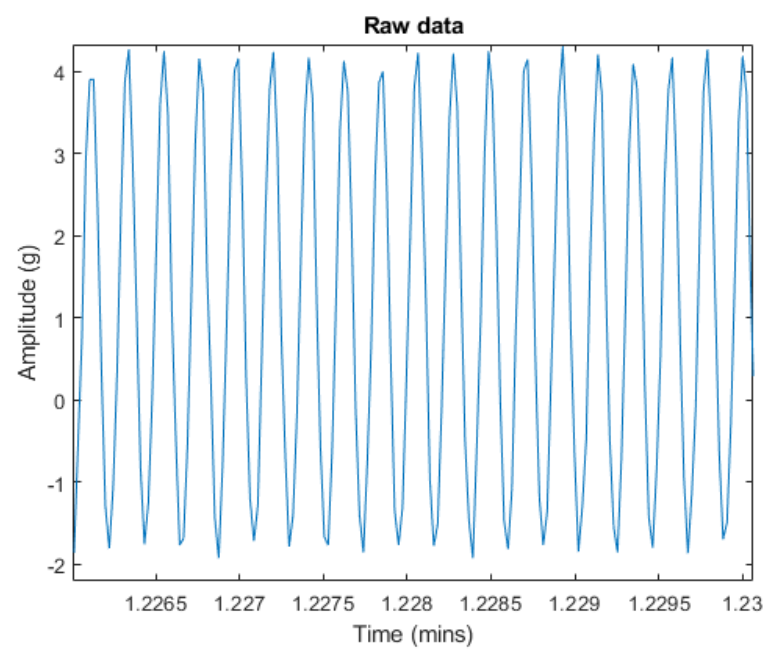

(b) A segment of the raw data

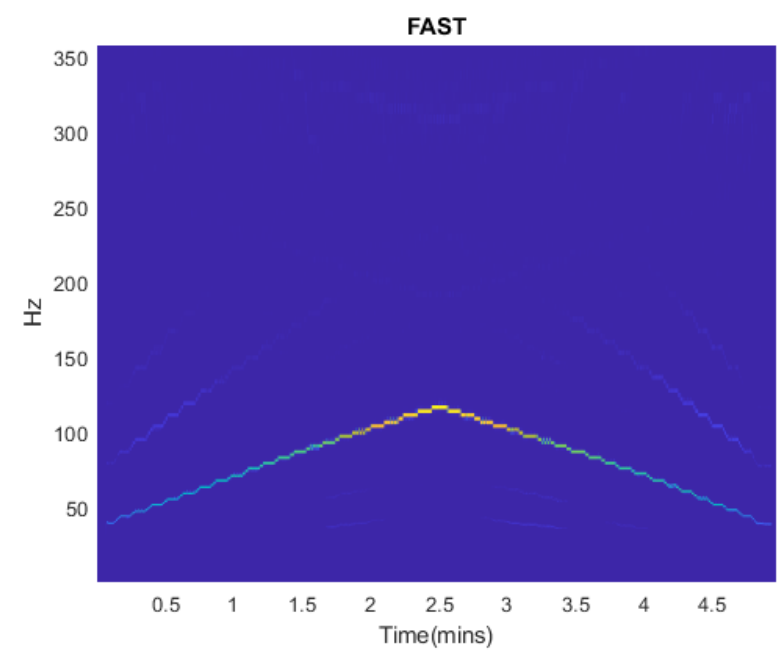

(d) FAST

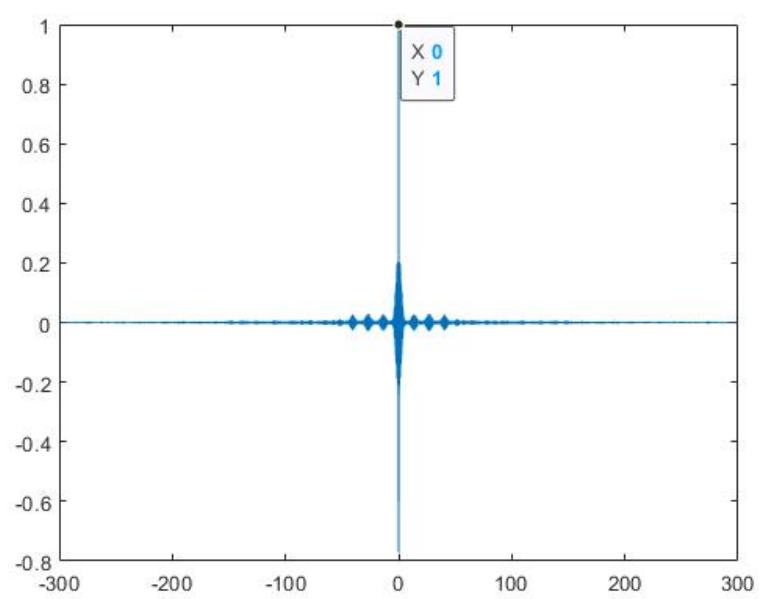

(f) Correlation between inverse of FAST and inverse of WSST 